\title{
Enhanced regeneration of large cortical bone defects with electrospun nanofibrous membranes and low-intensity pulsed ultrasound
}

\author{
LEYI HUANG ${ }^{1}$, YOUZHI CAI ${ }^{2}$, HONGHUA HU ${ }^{1}$, PENG GUO $^{3}$ and ZENGFENG XIN ${ }^{4}$
}

${ }^{1}$ Department of Orthopedic Surgery, The Fourth Affiliated Hospital, Medical College of Zhejiang University, Yiwu, Zhejiang 322000; ${ }^{2}$ Department of Orthopedic Surgery, The First Affiliated Hospital, Medical College of Zhejiang University, Hangzhou, Zhejiang 310009; ${ }^{3}$ Department of Orthopedic Surgery, The Eastern Hospital of The First Affiliated Hospital, Sun Yat-sen University, Guangzhou, Guangdong 510080; ${ }^{4}$ Department of Orthopedic Surgery, The Second Affiliated Hospital, Medical College of Zhejiang University, Hangzhou, Zhejiang 310009, P.R. China

Received March 1, 2016; Accepted February 24, 2017

DOI: $10.3892 / e t m .2017 .4565$

\begin{abstract}
Poly-L-lactic acid (PLLA) nanofibrous membranes are widely utilized for tissue regeneration. Low intensity pulsed ultrasound (LIPUS) has been considered as a feasible modality for bone union. The aim of the present study was to investigate the potential synergistic effect of LIPUS and PLLA electrospun nanofibrous membranes on large cortical bone defects in rabbits in vivo. The bilateral rabbit tibia defect model was constructed using 18 adult NZ rabbits and the defect sites were treated with the nanofibrous membranes combined with LIPUS or nanofibrous membranes alone. A total of 3 to 6 weeks after surgery, bone defect healing was evaluated radiologically and histologically. Radiographs demonstrated that nascent bone formation in the central part of the defect regions was only observed in the nanofibrous membrane plus LIPUS group, whereas the bone defects were not fully healed in the group treated with nanofibrous membrane alone. Histology analysis of the LIPUS-treated group indicated that bone formation was thicker and more mature in the center of the defect site of the nanofibrous membrane plus LIPUS group. However, no differences were detected in the spatial and temporal pattern of the newly formed bone. Furthermore, the bone scores in the nanofibrous membrane plus LIPUS group were significantly greater than the scores exhibited in the nanofibrous membrane group at 3 and 6 weeks after surgery, respectively $(\mathrm{P}<0.01)$. In conclusion, the PLLA electrospun nanofibrous membrane combined with LIPUS indicated the capacity to improve the
\end{abstract}

Correspondence to: Mr. Zengfeng Xin, Department of Orthopedic Surgery, The Second Affiliated Hospital, Medical College of Zhejiang University, 88 Jiefang Road, Hangzhou, Zhejiang 310009, P.R. China

E-mail: beckham_xin@hotmail.com

Key words: large cortical bone defect, low-intensity pulsed ultrasound, nanofibrous membrane formation of nascent bone in rabbits with tibia defects. Further studies are required to fully elucidate the cell ingrowth depths inside nanofibrous membranes with scanning electron microscopy and the molecular effects of LIPUS on integrin and fibronectin.

\section{Introduction}

Treating large bone defects remains a major challenge in clinical practice. Although many osteoconductive and osteoinductive filler materials have been used for bone defects (1,2), annually, $>800,000$ patients worldwide cannot receive this treatment but instead receive autologous bone grafts to treat their bone defects (3). The autologous bone grafting technique has been viewed as the 'gold standard' for its homogeneous bony tissue and efficacy; however, the method has limitations, including morbidity, limited resources and its association with complications, such as bleeding and wound problems $(4,5)$. On the basis of the data from the National Hospital Discharge Survey, the use of bone grafts decreased in the United States between 1992 and 2007, with a shift in preference from autogenous bone grafts to substitute bone grafts (6).

Previous studies have identified the structure of the nanofibers induced the proliferation of the mesenchymal stem cells and osteoblasts $(7,8)$. The structure and biological functions of poly-L-lactic acid (PLLA) electrospun nanofibers are similar to the natural extracellular matrix (ECM) of bone tissue and are superior to other biomaterials, due to their beneficial biocompatibility properties $(9,10)$. However, as the pores generated by electrospinning are within the range of the fiber diameters, the pores are not large enough for the cellular migration and tissue infiltration (11). Furthermore, the surface of PLLA is hydrophobic and lacks bioactive signals for cell recognition, which increases the difficulty for integrin receptors to identify binding sites $(12,13)$. Hence, further studies are required to overcome the disadvantages.

Approved by the Food and Drug Administration, low-intensity pulsed ultrasound (LIPUS) is considered as a non-invasive and feasible modality for the treatment of the delayed union and 
the nonunion of bone (14). A previous study has demonstrated that ultrasound exposure increased the porosity and permeability of the solid-state fabricated PLA foams (15). The findings are in agreement with results obtained from previous results from Guo et al (16). Moreover, other investigators have concluded that the bioeffect of LIPUS exposure was promoted via the integrin/FAK/MAPK pathway (17). Cell-matrix adhesions are predominantly mediated by the members of the integrin family (18). A previous study revealed that the conformation of proteins, which are composed of cell-matrix adhesions, changed and the cryptic-binding sites were exposed while mechanical force acted on the cell-matrix adhesions (19). Therefore, it seems reasonable that mechanical stresses, such as LIPUS acting on the cell-matrix adhesions, may expose the cryptic site.

The potential synergistic effect of LIPUS and PLLA electrospun nanofibers remains unclear. The present study investigated the hypothesis that the critical size of cortical bone defect filled with the PLLA electrospun nanofibrous membrane would acquire bone union with the aim of LIPUS treatment.

\section{Materials and methods}

Fabrication of PLLA electrospun nanofibers. The PLLA nanofibrous membrane was successfully produced by the polymer solution methodology as previously described $(20,21)$. The polymer solution was fabricated by dissolving PLLA (Thermo Fisher Scientific, Ltd., Waltham, MA, USA) in chloroform/ethanol (3:1, v/v). Then polymer was subsequently placed into a $5 \mathrm{ml}$ syringe connecting to a $50 \mathrm{~cm}$ Teflon tube (Bo Jie Co., Ltd, Huatan, Taiwan). The electrospinning solution was delivered with a flow rate of $1.0 \mathrm{ml} / \mathrm{h}$ via a syringe pump. The thickness of the PLLA was $2.0 \mathrm{~mm}$. All electrospun nanofibrous membranes were stored in the desiccators prior to use.

Animal experiment. The present study was approved by the Zhejiang Institutional Animal Care and Use Committee (Hangzhou, China). International laws and regulations for medical research with experimental animals were followed. A total of 18 adult male New Zealand White rabbits aged between 15 and 18 weeks and weighing $2.19 \pm 0.17 \mathrm{~kg}$, were randomly divided into two groups: The control group $(n=6)$ and the study group $(n=12)$. Rabbits were housed at $21-25^{\circ} \mathrm{C}, 60 \%$ humidity with a 12 h light/dark cycle. Rabbits had full access to dry food and water in individual cages without activity restriction. All rabbits were anesthetized by an intravenous injection with chloral hydrate $(280 \mathrm{mg} / \mathrm{kg}$; Tokyo Chemical Industry Co., Ltd., Tokyo, Japan) prior to surgery. The surgical procedures are described in detail in a previous study (21) and are briefly reported here. While the shaft of the tibia was exposed, a segmental defect (15-mm long and 5-mm wide) was created in the anteriolateral cortex of the tibia with an oscillating saw. This procedure was performed repeatedly in the bilateral tibias of each rabbit. After the bone defect was successfully administered in the anteromedial cortex of the bilateral proximal tibia, rabbits were treated differently according to the study protocol. Bone defects in rabbits were not induced in the control group. The nanofibrous membrane was cut into $15 \times 5 \mathrm{~mm}^{2}$ membrane specimens, which were used to fill the bone defects in the study group. All of the membrane specimens were press-fitted into the bone defect. The left tibias of the study group were treated with nanofibrous membranes, whereas the right tibias of rabbits were treated with nanofibrous membranes and LIPUS. The ultrasonic generator (Nexus; Hexin Biomedical Devices, Hangzhou, China) was employed with one ultrasonic transducer at a pulse frequency of $1.5 \mathrm{MHz}$, output intensity of $200 \mathrm{~mW} / \mathrm{cm}^{2}$ and a $50 \%$ duty cycle. Ultrasound gel was placed on the surface of the transducer and the surgical site when the LIPUS was applied. A total of 3 rabbits from the control group and the 6 from the study group were sacrificed at 3 and 6 weeks, respectively, for evaluation (Fig. 1).

Radiographic assessment. Rabbits were sacrificed via overdose of $200 \mathrm{mg} / \mathrm{kg}$ pentobarbital sodium administered intravenously via the marginal ear vein (Tokyo Chemical Industry Co., Ltd., Tokyo, Japan) at either 3 or 6 weeks. Tibiae from rabbits were harvested, fixed in $10 \%$ neutral formalin for 1 week at $4{ }^{\circ} \mathrm{C}$ and subsequently radiographed in the anteroposterior planes using X-ray apparatus (Kodak, Rochester, NY, USA).

Histological evaluation. Specimens were decalcified in $10 \%$ EDTA in $0.01 \mathrm{M}$ phosphate buffer and dehydrated with an ascending series of ethanol solutions for one week at $4^{\circ} \mathrm{C}$. Following decalcification and dehydration, the samples were embedded in paraffin. The area of interest was defined at $10-\mathrm{mm}$ proximally and distally to the center of the defect. Along the central portion of the defect in the anteromedial cortex, tibiae were cut horizontally on a microtome. Sections $(8-\mu \mathrm{m}$ thick) were stained with hematoxylin and eosin and examined under a light microscope (Olympus Corp., Tokyo, Japan). Histological analyses were performed at a primary magnification of x40 and a second magnification of $\mathrm{x} 200$ or $\mathrm{x} 400$.

Bone score. Bone scoring was applied to evaluate the degree of nascent bone formation during histological evaluation. The semiquantitative score from 0 (no mineralized bone) to 4 (complete bridging of the defect with mineralized bone) was used in the present study, as described previously (22). The other score values were defined as follows: 1 , few and isolated centers of ossification; 2, increased ossification with discontinuous novel bone formation; and 3, notable but incomplete bridging of the defect (22). Scoring was evaluated by at least two investigators. The score was added to give a cumulative sum to estimate the overall bone formation in the defect at a given time. Bone scores were compared among all groups by at least two persons with no knowledge of the groups being graded.

Statistical analysis. Statistical analysis was performed using GraphPad Prism 6.0 software package (GraphPad Software, Inc., La Jolla, CA, USA). The bone formation ratio is expressed as the mean \pm standard deviation and the normality of the distribution was tested. For multiple comparisons among groups, differences were analyzed by one-way analysis of variance followed by Tukey's post-hoc test. $\mathrm{P}<0.01$ was considered to indicate a statistically significant difference.

\section{Results}

Excluded results. One animal in the control group was sacrificed due to a postoperative hematoma and 

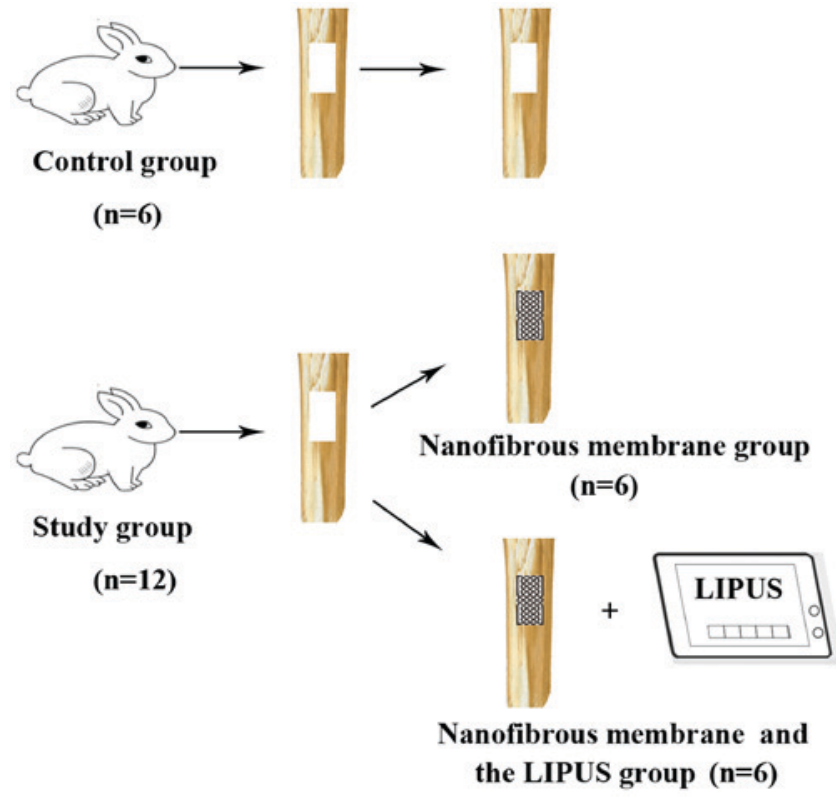

Figure 1. Flow chart of the study. Defects in the tibiae of rabbits were not treated (control group), treated with the poly-L-lactic acid nanofibrous membrane (left tibiae of rabbits) or treated with the poly-L-lactic acid nanofibrous membrane plus LIPUS (right tibiae of rabbits). LIPUS, low intensity pulsed ultrasound.

secondary infection. All other animals recovered from the bilateral surgery and completed the study without any complications.

$X$-ray evaluation. No signs of gross adverse reactions or infection according to the macroscopic examination of the proximal tibia at harvest were observed. The representative conventional $\mathrm{X}$-ray photographs of the tibia cortex are shown in Fig. 2. All of the control group and the study groups showed that bone healing did not occur completely 3 weeks after surgery. Small areas of mineralization scattered at the bone defects margin were detectable in the nanofibrous membrane group and nanofibrous membrane plus LIPUS group. Conversely, the bone defects filled with nanofibrous membranes and treated with LIPUS demonstrated increased bone formation compared with the nanofibrous membrane group. However, in the control group, the defect sites indicated no bone ingrowth. A total of 6 weeks after surgery, novel bone formation in the central part of the defect regions was observed only in the nanofibrous membrane plus LIPUS group. The bone defect was not fully healed in the nanofibrous membrane group and the defect was filled with radio-opaque tissue, with the exception of the central region. The control group showed no areas of mineralization.

Histological evaluation. Microscopically, the residues of the nanofibrous membranes were visible at 3 and 6 weeks; this is consistent with previous findings (21). The results indicated that LIPUS did not alter the resorption rate of nanofibrous membranes according to the sections. The aligned fibers, which covered the bone defect site, guided the newly formed bone from the edge of the gap to the other edge. In addition, the amount of newly formed bone and connective tissue in the study group was different from the control group. Increased volumes of newly formed cortical bones were indicated in the nanofibrous membrane group and nanofibrous membrane plus LIPUS group (Fig. 3).

The formation of novel bone adjacent to the defect margin was observed in all groups except for the control group at 3 weeks. The bone defect site of the control group was filled with connective tissue. Novel bone formation was consistently identified where the nanofibrous membranes were in direct contact with the defect margin. Combined with nanofibrous membranes, LIPUS treatment increased the amount of newly formed bone when compared with nanofibrous membrane alone. An obvious increase in nascent bone formation was observed with ultrasound treatment (Fig. 3). Based on these findings, defects treated with nanofibrous membranes plus LIPUS demonstrated thicker bone-like tissue.

At 6 weeks, in the nanofibrous membrane plus LIPUS group, abundant mature lamellar bone was observed running through the margins of the bone defect via the nanofibrous membrane. Nascent bone formation was observed primarily in proximity with the nanofibrous membranes. Compared with the nanofibrous membrane group, the bone formation of the nanofibrous membrane plus LIPUS group was thicker and more mature in the center of the defect site. However, the spatial and temporal pattern of novel bone formation indicated no difference between the nanofibrous membrane plus LIPUS group and the nanofibrous membrane alone. Newly-formed capillaries and osteoblasts were also observed in the study group, which are responsible for the formation of bone. Newly formed bone, which appeared to be spongy, and connective tissue were scattered in the defect sites of the control group. Limited mature lamellar bone was visible in the control group (Fig. 3).

Bone score. Significant differences in bone scores were determined between the nanofibrous membrane plus LIPUS group and the nanofibrous membrane group at 3 and 6 weeks, respectively ( $\mathrm{P}<0.01$; Figs. 4 and 5 , respectively). The nanofibrous membrane plus LIPUS group exhibited a significantly greater bone score when compared with the nanofibrous membrane group $(\mathrm{P}<0.01$; Figs. 4 and 5).

\section{Discussion}

The healing of large bone defects remains a clinical challenge. The majority of studies have focused on the biological, material or mechanical factors that are applied for the treatment of bone defects. As it is difficult to create enough macropores for cellular migration using the electrospun PLLA nanofibrous membrane and the surface of the PLLA lacks bioactive signals for cell recognition, we believe the LIPUS may be utilized to overcome these disadvantages.

The present study evaluated the efficacy of LIPUS combined with the cell-impermeable PLLA nanofibrous membrane on the healing of large cortical bone defects. The present findings suggested that LIPUS was able to increase cell ingrowth and expose the cryptic site when acting on cell-matrix adhesions. Furthermore, the present study demonstrated that LIPUS improved the efficacy of PLLA. A predominant challenge of the widespread use of electrospun nanofibers has been their innate hydrophobic nature and the difficulty in creating enough macropores for cellular migration $(11,13)$. The pores generated 


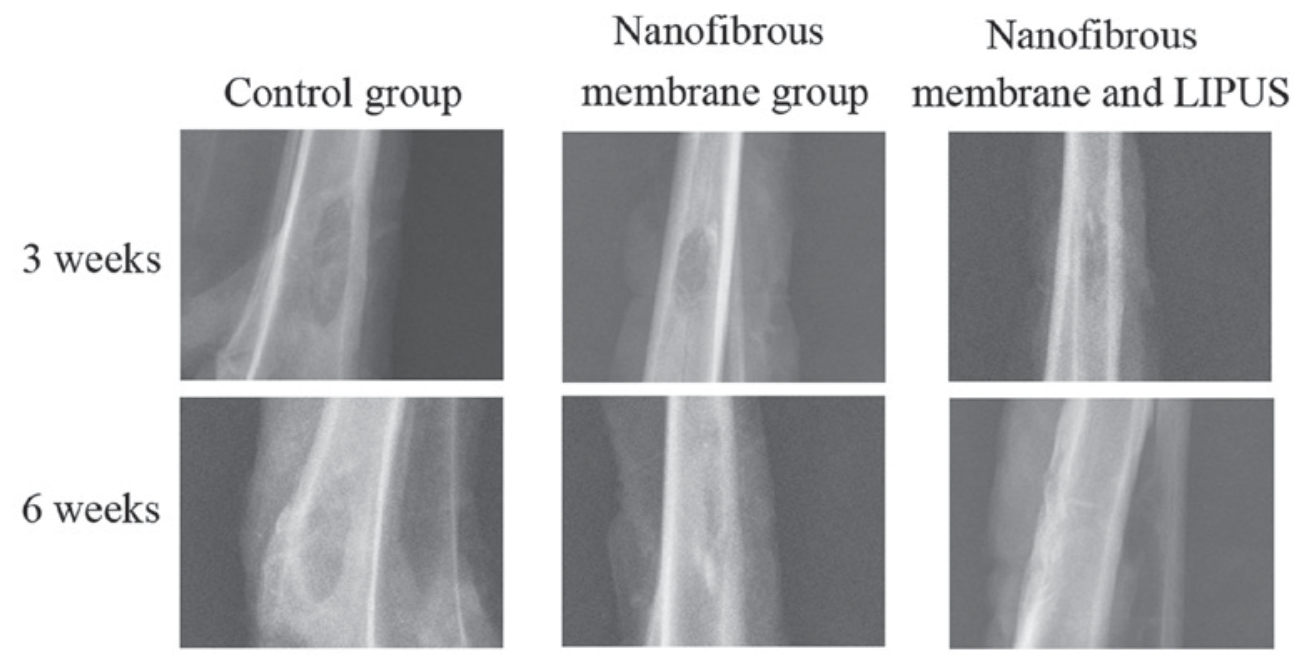

Figure 2. Radiographic appearance of defects in the tibiae of rabbits at 3 and 6 weeks, respectively. LIPUS, low intensity pulsed ultrasound.
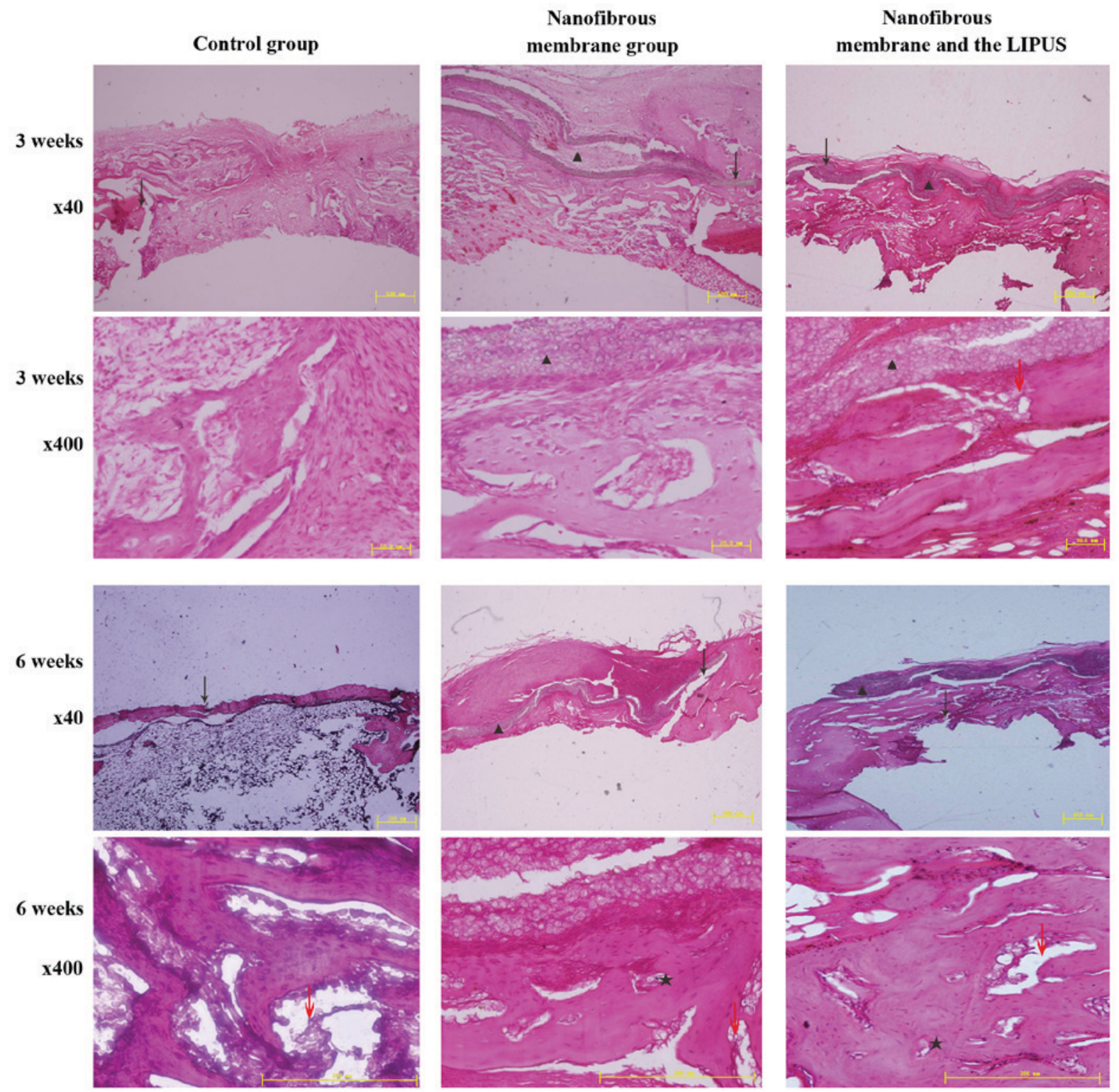

Figure 3. Histological slices of the tibiae defects in control and treatment groups at 3 and 6 weeks, respectively. Black arrows represent defect margins, red arrows represent blood vessels, black triangles represent nanofibers and black stars represent osteoblasts. Scale bar, $50 \mu \mathrm{m}$ or $200 \mu \mathrm{m}$ at a magnification of x400. Scale bar, $500 \mu \mathrm{m}$ at a magnification of x40. LIPUS, low intensity pulsed ultrasound. 


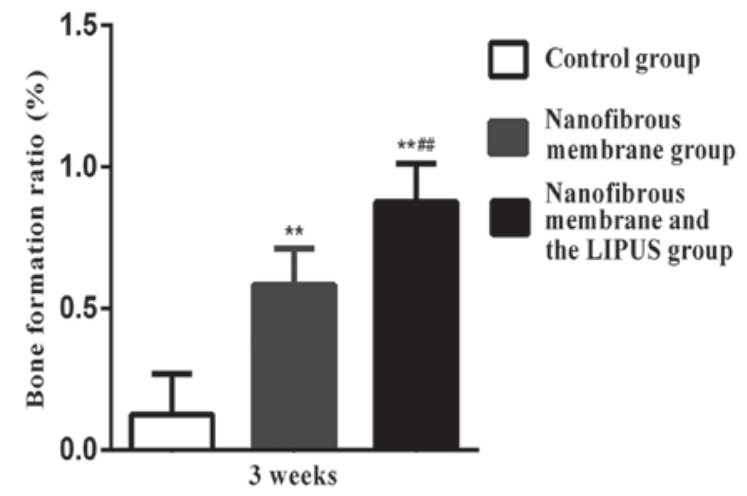

Figure 4. Bone formation ratio at 3 weeks. LIPUS, low intensity pulsed ultrasound. The nanofibrous membrane plus LIPUS group exhibited a significantly greater bone score compared with the nanofibrous membrane and control groups. Data are presented as the mean \pm standard deviation. ${ }^{* *} \mathrm{P}<0.01$ vs. control group; ${ }^{\#} \mathrm{P}<0.01$ vs. nanofibrous membrane group. LIPUS, low intensity pulsed ultrasound.

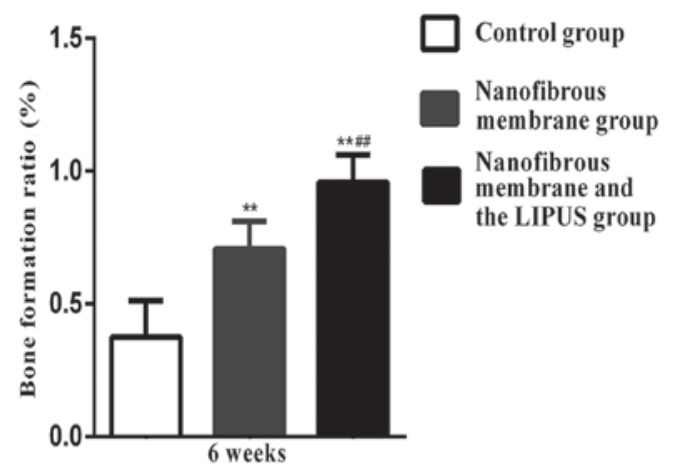

Figure 5. Bone formation ratio at 6 weeks. LIPUS, low intensity pulsed ultrasound. The nanofibrous membrane plus LIPUS group exhibited a significantly greater bone score when compared with the nanofibrous membrane and control groups. Data are presented as the mean \pm standard deviation. ${ }^{* *} \mathrm{P}<0.01$ vs. control group; ${ }^{\# \#} \mathrm{P}<0.01$ vs. nanofibrous membrane group. LIPUS, low intensity pulsed ultrasound.

by the electrospinning are approximately the same order of the fiber sizes $(11,23)$. A previous study hypothesized that the surrounding fibers were dynamically moved by cells entering the pores (24). Few studies have explored this hypothesis and further investigation is required.

When implanted material is placed in the bone defect, cells from the surrounding tissues interact with the membrane material in a process, which is mediated by the protein that is adsorbed to the surface (25). Mesenchymal stem cells are able to detect and migrate along the surface of the membrane. In the present study, thicker and more mature bone formed in the bone defect site adjacent to the membrane in the nanofibrous membrane plus LIPUS group. Detection of mesenchymal stem cells infiltrating the membrane was limited; however, the aligned fibers guided the newly formed bone from the edge of the gap to the center of the defect. Previous investigation has demonstrated that aligned nanofibers increased cell migration along the direction of fiber orientation, but did not influence osteogenic differentiation (26). Therefore, this suggests that the efficacy of bone formation in the present study may be attributed to LIPUS rather than the PLLA nanofibrous membrane.
Although the morphology and cell viability were not measured by scanning electron microscopy, the findings of the present study suggested that LIPUS may increase cell ingrowth into porous membranes. Previous results have indicated that LIPUS treatment accelerated bone ingrowth into the porous tricalcium phosphate bioceramic scaffold (27) and increased cell ingrowth into the pores of the three-dimensional silicon carbide scaffold (28). These findings suggest that the increased bone formation observed in the PLLA nanofibrous membrane plus LIPUS group in the present study may be attributed to the capacity for LIPUS to increase cell ingrowth into porous nanofibrous membranes. Further investigations and scanning electron microscopy should be performed to clarify the cell ingrowth depths inside the nanofibrous membrane.

The findings obtained from the present study indicated that only the nanofibrous membrane plus LIPUS group displayed near-complete regeneration of the cortical bone 6 weeks after surgery. Newly formed bone was visible in the center of the bone defect site in contact with the nanofibrous membrane in the presence or absence of LIPUS. Therefore, the nanofibrous membrane acted as the bridge that guided the osteoblasts or the progenitors to the bone defect site. The results suggested that the nanofibrous membrane improved the bone formation and induced osteogenic differentiation. The present findings was in accordance with the findings presented by Kolambkar et al (26), who reported aligned nanofiber meshes with increased cell migration along the direction of fiber orientation.

Compared with the nanofibrous membrane group, the bone formation of the nanofibrous membrane plus LIPUS group was thicker and more mature; however, the spatial and temporal pattern of the newly formed bone suggested that there was no difference between these groups. It predicted that LIPUS increased bone formation rather than accelerated the bone healing. Based on the present findings, we believe the efficacy of LIPUS first acted on the integrin, which indicates that this is the starting point of mechanosensing.

Electrospun nanofibers mimic the native extracellular tissue structures and exhibit suitable biocompatibility (24). In spite of this, aligned nanofibers may not influence osteogenic differentiation or provide a limited contribution to the process. The mechanisms involved in the limited influence of aligned nanofibers and osteogenic differentiation remain unclear. However, the lack of bioactive sites for cell recognition and could cause difficulties for cells interacting with the extracellular matrix $(12,13)$. When cells attach to extracellular tissue structures, the process by which cells probe the environment and translate mechanical signal into biochemical signals is called mechanosensing (29). During the process, integrins, which initiate mechanosensing, are necessary elements in the focal adhesions for the majority of mechanosensing models $(30,31)$. The cytoskeletal force generated by myosin II combines with the ECM stiffness triggers the $\alpha 5 \beta 1$ integrin switch (31). Subsequently, the $\alpha 5 \beta 1$ integrin is able to mediate downstream biochemical signals that control cell fate and adhere to a fibronectin substrate. Once the fibronectin interacts with integrin receptors, polymerization of fibronectin initiates and the cryptic site for fibronectin-fibronectin polymerization is exposed (19). The process of the conformation change may expose cryptic sites to overcome the drawbacks of nanofibrous membranes. 
Notably, a previous study indicated that LIPUS stimulation may activate $\alpha 5 \beta 1$ integrin and induce a significant upregulation of marker proteins as the representation of the osteoblasts (32). Furthermore, the integrin was also highly activated under fluid flow stimulation as well as LIPUS (33). A recent study investigated the effect of LIPUS stimulation on mesoangioblasts and demonstrated that LIPUS may induce a conformational change in $\beta 1$ integrin to the active form (34).

In conclusion, the present study demonstrated that the PLLA electrospun nanofibrous membranes combined with the LIPUS improved the formation of novel bone in rabbit tibiae defects. As the critical size of the cortical bone defect acquired near-complete regeneration of cortical bone tissue, PLLA electrospun nanofibrous membranes combined with the LIPUS may be a novel potential therapy for the application of bone tissue engineering. Moreover, further studies are required to fully investigate the cell ingrowth depths inside the nanofibrous membrane with scanning electron microscopy and the molecular effects of LIPUS on integrin and fibronectin.

\section{References}

1. Bhatt RA and Rozental TD: Bone graft substitutes. Hand Clin 28: 457-468, 2012.

2. Kurien T, Pearson RG and Scammell BE: Bone graft substitutes currently available in orthopaedic practice: The evidence for their use. Bone Joint J 95-B: 583-597, 2013.

3. Wang G, Yang H, Li M, Lu S, Chen X and Cai X: The use of silk fibroin/hydroxyapatite composite co-cultured with rabbit bone-marrow stromal cells in the healing of a segmental bone defect. J Bone Joint Surg Br 92: 320-325, 2010.

4. Bucholz RW: Nonallograft osteoconductive bone graft substitutes. Clin Orthop Relat Res: 44-52, 2002.

5. Van der Stok J, Van Lieshout EM, El-Massoudi Y, Van Kralingen GH and Patka P: Bone substitutes in the Netherlandsa systematic literature review. Acta Biomater 7: 739-750, 2011.

6. Kinaci A, Neuhaus V and Ring DC: Trends in bone graft use in the United States. Orthopedics 37: e783-e788, 2014.

7. Woo KM, Jun JH, Chen VJ, Seo J, Baek JH, Ryoo HM, Kim GS, Somerman MJ and Ma PX: Nano-fibrous scaffolding promotes osteoblast differentiation and biomineralization. Biomaterials 28: 335-343, 2007

8. Xin X, Hussain M and Mao JJ: Continuing differentiation of human mesenchymal stem cells and induced chondrogenic and osteogenic lineages in electrospun PLGA nanofiber scaffold. Biomaterials 28: 316-325, 2007.

9. Dong S, Sun J, Li Y,Li J, Cui W and Li B: Electrospun nanofibrous scaffolds of poly (L-lactic acid)-dicalcium silicate composite via ultrasonic-aging technique for bone regeneration. Mater Sci Eng C Mater Biol Appl 35: 426-433, 2014.

10. Stevens MM and George JH: Exploring and engineering the cell surface interface. Science 310: 1135-1138, 2005.

11. Jang JH, Castano O and Kim HW: Electrospun materials as potential platforms for bone tissue engineering. Adv Drug Deliv Rev 61: 1065-1083, 2009.

12. Schofer MD, Boudriot U, Bockelmann S, Walz A, Wendorff JH, Greiner A, Paletta JR and Fuchs-Winkelmann S: Effect of direct RGD incorporation in PLLA nanofibers on growth and osteogenic differentiation of human mesenchymal stem cells. J Mater Sci Mater Med 20: 1535-1540, 2009.

13. Liu W, Zhan J, Su Y, Wu T, Wu C, Ramakrishna S, Mo X, Al-Deyab SS and El-Newehy M: Effects of plasma treatment to nanofibers on initial cell adhesion and cell morphology. Colloids Surf B Biointerfaces 113: 101-106, 2014.

14. Mehta S, Long K, DeKoven M, Smith E and Steen RG: Low-intensity pulsed ultrasound (LIPUS) can decrease the economic burden of fracture non-union. J Med Econ 18: 542-549, 2015 .
15. Guo G, Lu L, Ji H, Ma Y, Dong R, Tu J, Guo X, Qiu Y, Wu J and Zhang D: Low intensity pulse ultrasound stimulate chondrocytes growth in a 3-D alginate scaffold through improved porosity and permeability. Ultrasonics 58: 43-52, 2015.

16. Guo G, Ma Q, Zhao B and Zhang D: Ultrasound-assisted permeability improvement and acoustic characterization for solid-state fabricated PLA foams. Ultrason Sonochem 20: 137-143, 2013.

17. Sato M, Nagata K, Kuroda S, Horiuchi S, Nakamura T, Karima M, Inubushi T and Tanaka E: Low-intensity pulsed ultrasound activates integrin-mediated mechanotransduction pathway in synovial cells. Ann Biomed Eng 42: 2156-2163, 2014.

18. Hynes RO: Integrins: Bidirectional, allosteric signaling machines. Cell 110: 673-687, 2002.

19. Kadler KE, Hill A and Canty-Laird EG: Collagen fibrillogenesis: Fibronectin, integrins, and minor collagens as organizers and nucleators. Curr Opin Cell Biol 20: 495-501, 2008.

20. Zhang Y, Ouyang H, Lim CT, Ramakrishna S and Huang ZM: Electrospinning of gelatin fibers and gelatin/PCL composite fibrous scaffolds. J Biomed Mater Res B Appl Biomater 72: 156-165, 2005.

21. Cai YZ, Wang LL, Cai HX, Qi YY, Zou XH and Ouyang HW: Electrospun nanofibrous matrix improves the regeneration of dense cortical bone. J Biomed Mater Res A 95: 49-57, 2010.

22. Sarkar MR, Augat P, Shefelbine SJ, Schorlemmer S, Huber-Lang M, Claes L, Kinzl L and Ignatius A: Bone formation in a long bone defect model using a platelet-rich plasma-loaded collagen scaffold. Biomaterials 27: 1817-1823, 2006.

23. Zhang YZ, Ouyang H, Lim CT, Ramakrishna S and Huang ZM: Electrospinning of gelatin fibers and gelatin/PCL composite fibrous scaffolds. J Biomed Mater Res B Appl Biomater 72: 156-165, 2005.

24. Bhattarai SR, Bhattarai N, Yi HK, Hwang PH, Cha DI and Kim HY: Novel biodegradable electrospun membrane: Scaffold for tissue engineering. Biomaterials 25: 2595-2602, 2004.

25. Zhu XD, Fan HS, Xiao YM, Li DX, Zhang HJ, Luxbacher T and Zhang XD: Effect of surface structure on protein adsorption to biphasic calcium-phosphate ceramics in vitro and in vivo. Acta Biomater 5: 1311-1318, 2009.

26. Kolambkar YM, Bajin M, Wojtowicz A, Hutmacher DW, García AJ and Guldberg RE: Nanofiber orientation and surface functionalization modulate human mesenchymal stem cell behavior in vitro. Tissue Eng Part A 20: 398-409, 2014.

27. Hui CF, Chan CW, Yeung HY, Lee KM, Qin L, Li G, Leung KS, $\mathrm{Hu}$ YY and Cheng JC: Low-intensity pulsed ultrasound enhances posterior spinal fusion implanted with mesenchymal stem cells-calcium phosphate composite without bone grafting. Spine (Phila Pa 1976) 36: 1010-1016, 2011.

28. Wu L, Lin LJ and Qin YX: Enhancement of cell ingrowth, proliferation, and early differentiation in a three-dimensional silicon carbide scaffold using low-intensity pulsed ultrasound. Tissue Eng Part A 21: 53-61, 2015.

29. Schiller HB and Fässler R: Mechanosensitivity and compositional dynamics of cell-matrix adhesions. EMBO Rep 14: 509-519, 2013.

30. Lv H, Li L, Sun M, Zhang Y, Chen L, Rong Y and Li Y: Mechanism of regulation of stem cell differentiation by matrix stiffness. Stem Cell Res Ther 6: 103, 2015.

31. Friedland JC, Lee MH and Boettiger D: Mechanically activated integrin switch controls alpha5betal function. Science 323: 642-644, 2009.

32. Watabe H, Furuhama T, Tani-Ishii N and Mikuni-Takagaki Y: Mechanotransduction activates $\alpha 5 \beta 1$ integrin and PI3K/Akt signaling pathways in mandibular osteoblasts. Exp Cell Res 317: 2642-2649, 2011.

33. Vaughan TJ, Mullen CA, Verbruggen SW and McNamara LM: Bone cell mechanosensation of fluid flow stimulation: A fluid-structure interaction model characterising the role integrin attachments and primary cilia. Biomech Model Mechanobiol 14: 703-718, 2015.

34. Bernal A,Pérez LM, De Lucas B, Martín NS, Kadow-Romacker A, Plaza G, Raum K and Gálvez BG: Low-intensity pulsed ultrasound improves the functional properties of cardiac mesoangioblasts. Stem Cell Rev 11: 852-865, 2015. 